\title{
Variations
}

Variations

Revue internationale de théorie critique

$22 \mid 2019$

Gorz, l'intempestif

\section{Habermas, philosophe allemand de la restauration française?}

Alexander Neumann

\section{OpenEdition}

Journals

Édition électronique

URL : http://journals.openedition.org/variations/1061

DOI : 10.4000/variations. 1061

ISSN : 1968-3960

Éditeur

Les amis de Variations

Référence électronique

Alexander Neumann, « Habermas, philosophe allemand de la restauration française? », Variations [En ligne], 22 | 2019, mis en ligne le 04 mars 2019, consulté le 19 avril 2019. URL : http:// journals.openedition.org/variations/1061; DOI : 10.4000/variations.1061

Ce document a été généré automatiquement le 19 avril 2019

Les ami•e•s de Variations 


\title{
Habermas, philosophe allemand de la restauration française?
}

\author{
Alexander Neumann
}

1 Karl Marx avait salué le philosophe Emmanuel Kant comme étant le théoricien politique de la révolution française ${ }^{1}$, le nommant aussi le grand maître, le Meister Kant. Il serait aujourd'hui possible de comprendre Jürgen Habermas comme un théoricien allemand de la restauration française. Dans l'année qui marque le 90ème anniversaire du penseur, il s'agit d'une lecture critique qui promet d'enrichir un peu l'image conformiste que cultive la presse française, Le Monde en tête (23/02/18). Après avoir milité pendant plus de 60 ans dans le SPD, le parti social-démocrate, Habermas soutient et célèbre aujourd'hui le président Emmanuel Macron comme un personnage "exceptionnel", "fascinant", et même "éblouissant" (L'Obs, 26/10/2017), après avoir publiquement soutenu sa campagne électorale. Cette ode quelque peu acritique n'est pas une expression isolée, mais s'inscrit dans un parcours philosophico-politique émaillé de prises de positions discutables, depuis l'hitlérisme juvénile jusqu'à la défense du bombardement de la Serbie, en passant par le rejet de la gauche socialiste, la condamnation du droit naturel de la révolution française, l'antimarxisme militant ou encore l'enterrement explicite de l'Ecole de Francfort. Le tout se confirme successivement dans son ralliement au club de réflexion de Dominique Strauss Kahn², au projet européen de Giscard D'Estaing et de Jacques Chirac, et enfin dans son soutien public au candidat Emmanuel Macron, à travers un meeting berlinois commun qui s'est tenu le 16 mas 2017 à la Hertie School of Governance. La question est de savoir dans quelle mesure ce penchant pour des positions de droite, de la part d'un intellectuel politique aussi exposé que Habermas, imprime ses théorisations successives, qui n'ont jamais prétendu à la neutralité. Dans une biographie pointue, Stefan MüllerDoohm nous présente ce philosophe engagé comme un cogneur dont la stature se forme à travers des querelles et des polémiques de toute sorte. ${ }^{3}$ Habermas ressemble à John Wayne, qui tire d'abord et discute après, comme il l'a lui-même avoué publiquement en 2001, ce que le biographe souligne d'un air amusé. L'intellectuel est plus vivace, impulsif et contradictoire que certains apologues, hagiographes ou courtisans français ne peuvent l'admettre. Je ne pense pas que l'homme Habermas, fort de l'expérience d'une vie, d'une somme colossale de distinctions, de prix et d'honneurs, ait besoin d'une laudatio de plus. Il 
est tout à fait possible qu'il puisse déceler une marque de reconnaissance dans une critique argumentée, alors que les vils flatteurs et les faux humbles furent si bien caractérisés par Kant dans son éthique politique, les identifiant à un esprit de grouillot, indigne du point de vue de la citoyenneté. ${ }^{4}$

2 Ici, il s'agit de prendre au sérieux le rôle de Habermas en tant qu'intellectuel politique de premier plan, statut que cet auteur revendique absolument, à partir d'une confrontation de ses principales interventions et conceptualisations qui vont de 1939 à 2019.

\section{Habermas entre Hitler et Horkheimer}

3 Commençons par le début, l'erreur de jeunesse de Jürgen Habermas, né en 1929, celle qui le pousse à se distinguer dès ses dix ans dans la section puberté des Jeunesses hitlériennes, le Jungvolk, au début de la guerre mondiale. Il avance ici vers le statut de chef (Führer), ce qui rend superflu une adhésion à la section supérieure des Jeunesses hitlériennes selon la règle alors en vigueur. ${ }^{5}$ Seul les adhérents zélés ont été promus chefs, bien entendu, leur offrant le choix de rester dans leur section initiale ou de passer à la section suivante de la Hitlerjugend. Contrairement à une impression répandue, tout le monde n'était pas enrôlé; le taux d'organisation de la Hitlerjugend avoisina les $60 \%$ d'une classe d'âge, ce qui signifie que $40 \%$ des jeunes n'en furent jamais partie. ${ }^{6}$ L'expérience nazie de Habermas prend fin lorsqu'il a 16 ans, grâce à la victoire de l'Armée rouge à Berlin, après la libération de Tunis, Paris et Rome par les alliés. L'étendue du désastre le fait réfléchir, il regrette alors son militantisme, mais il aura d'abord du mal à rompre avec le discours implicite des intellectuels nazis, après la guerre.

4 Au début des années 1950, la thèse doctorale de Habermas sur Schelling fut dirigée par Erich Rothacker, qui n'est pas un inconnu en Allemagne. Le professeur fut nommé pendant la guerre, il est un ancien membre haut placé du parti nazi, signataire de l'appel des 300 universitaires de mars 1933 en faveur d'Hitler, co-directeur en 1934 du volume 4 d'une Introduction à la philosophie qui est consacrée à la théorie des races. Rothacker dirigea un département du Ministère de la propagande sous la responsabilité du Dr. Goebbels, où il prit personnellement en charge l'action qui consista à brûler les livres interdits aux côtés des Jeunesses hitlériennes ${ }^{7}$. Voilà quelques indices qui éclairent l'environnement universitaire du jeune docteur Habermas et sa difficulté à se défaire de l'héritage discursif des anciens intellectuels nazis, dans un cadre républicain de l'Allemagne de l'Ouest qui se veut antifasciste.

5 A la même époque, Habermas publie un article remarqué en défense de la philosophie de Martin Heidegger, qui fut l'un des théoriciens prisés par les Jeunesses hitlériennes, dans le quotidien de droite qui est imprimé en lettres gothiques, la $F A Z$ (25/7/1953). L'étudiantchercheur tente ici de séparer complètement l'engagement politique de Heidegger de ses écrits théoriques. Ses cours philosophiques de 1935 venaient pourtant d'être révélées, signalant des références à un discours national-socialiste explicite ${ }^{8}$. Habermas défend néanmoins la thèse d'un découplage total, entre le philosophe et l'idéologue. Il va réitérer cette interprétation en 1987 encore, lorsqu'il préface la traduction allemande d'un livre de Victor Farias qui a permis de préciser l'engagement nazi d'Heidegger, membre du parti jusqu'au dernier moment. ${ }^{9}$ Le philosophe avait publiquement juré allégeance au Führer, fait écarter Husserl grâce aux purges antisémites, et il est ensuite resté membre du Nsdap jusqu'en 1945. Sa distanciation envers Hitler à partir de 1934 est toute relative, en réalité, car il ne s'agit nullement d'une rupture avec le national-socialisme, mais d'une critique 
du caractère insuffisamment radical de son application. Comme Farias l'a mis en relief, Heidegger soutint la ligne idéologique implacable du courant des SA, au sein du parti, dont les chefs furent éliminés par Hitler en 1934 lors de la nuit des longs couteaux. ${ }^{10}$ Le chef des SA, Röhm, avait dénoncé la fin du processus nationaliste-révolutionnaire, à travers une campagne dont le point culminant et le coup d'arrêt fut un discours contre le maintien du "vieux système" et contre le manque d'entrain d'Hitler, daté du 18 avril 1934. ${ }^{11}$ Le fait est que Heidegger démissionne de son poste de recteur le 26 avril 1934, une semaine plus tard, sans pour autant quitter le parti. Sa critique se dirige contre un pouvoir établi, qui aurait renoncé à la révolution nationale authentique, au nom du discours théorique le plus fanatique de l'extrême droite, dont l'antisémitisme militant ne fut jamais démenti. Les recherches scientifiques récentes ont mis en évidence les jeux de correspondance, entre l'idéologie antisémite et le discours philosophique d'Heidegger, qui a lui-même souligné cette association dans ses Cahiers noirs à propos de 'la juiverie mondiale ${ }^{12}$. A un autre moment, il divague au sujet d'une prétendue '"prédestination particulière de la communauté juive pour la criminalité planétaire", etc. ${ }^{13}$ Après la guerre, Karl Jaspers est chargé d'évaluer le rôle et la responsabilité de Heidegger durant la période 1933 à 45, à sa propre demande, ce qui donne lieu à un rapport qui souligne sa "pensée dictatoriale", entrainant l'interdiction d'enseigner en 1946. Herbert Marcuse a bien formulé le rapport de Heidegger de la théorie à la pratique, en 1947 : Nous ne pouvons opérer une séparation entre le philosophe et la personne de Heidegger ${ }^{14}$ Corriger le discours nazi d'Heidegger par la pensée d'Heidegger, comme le tenta maladroitement Habermas, est une entreprise vouée à l'échec. Habermas n'est pas intervenu dans le débat public sur les Cahiers noirs, ce qui indique qu'il a compris l'inanité de pareille approche qui voulait sauver Heidegger contre Heidegger, à une époque où le jeune auteur se classe encore parmi les heideggériens orthodoxes. ${ }^{15}$

Dans un premier temps, Habermas avait ainsi cherché à écarter le passif nazi du philosophe sur le plan politique, sans rompre explicitement avec son lourd soubassement discursif. Il n'est pas le seul qui erre face à l'horreur à cette époque, ce qui n'enlève rien au problème. Lorsqu'il prépare sa thèse de doctorat, L'absolu et l'histoire (1954), Habermas adhère encore sans réserve à cet héritage, puisqu'il y valorise le penseur de la restauration, Schelling, dans le cadre général du discours philosophique d'Heidegger. ${ }^{16}$ Cette approche ne contredit pas tellement la propagande nazie passée, qui valorisa le contre-révolutionnaire Schelling à l'encontre de l'esprit de la révolution française et des hégéliens de gauche - dont Marx est issu. Pareille posture se manifeste déjà dans les cours de Heidegger de 1936, où Schelling est loué comme une sorte de prophète d'une onthéologie, une ontologie théologique qui chante le devenir de l'empire. Exit le jeune Schelling qui discuta passionnément la révolution française avec Hegel lorsqu'ils étaient tous deux étudiants. Voici le Schelling tardif, impérial et anti-démocratique, qui fut en effet chargé de son vivant de combattre les effets subversifs de la dialectique hégélienne à l'Université. Un peu comme Heidegger en son temps, qui écarte Husserl et les phénoménologues juifs de l'Université de Fribourg. Des livres de poche à grand tirage, aujourd'hui oubliées, avaient assuré la diffusion propagandiste de la position nazie en faveur du Schelling de la restauration impériale, jusqu'aux années années $1940^{17}$. Notons que Habermas n'a pas souhaité publier sa thèse sur Schelling, jusqu'à aujourd'hui, en dépit de la coutume allemande qui attend qu'un doctorat soit édité en tant que livre. 
7 Enfin, la période post-nazie aux héritages troubles de Habermas cesse vers 1956, lorsqu'il commence à suivre les cours d'Adorno et Horkheimer à l'Institut de recherche sociale à Francfort, grâce à une bourse.

8 Cette rencontre permet à Habermas de découvrir les écrits de Karl Marx, que son directeur de thèse Rothacker avait littéralement fait brûler. Habermas va, lui, s'approcher du marxisme social-démocrate de l'époque, avant d'adhérer au parti, le SPD. Si Adorno remarque les talents intellectuels d'Habermas, qui s'avère capable de discuter les thèmes de l'idéalisme allemand, son rôle dans les grandes recherches de l'Institut s'avère particulièrement faible. Il touche un salaire d'assistant pour avoir participé à une seule enquête sur la politisation des étudiants, à coté de deux autres enquêteurs, et qui fut encadrée par les professeurs Horkheimer et Friedeburg. Face à la dissociation à peu près complète dont fait alors preuve Habermas, entre ses réflexions philosophiques personnelles et les recherches empiriques en sciences sociales, dissociation mal colmatée par une sorte de marxisme spéculatif récemment assimilé, Horkheimer refuse que l'Institut de Francfort garantisse une habilitation à diriger des recherches, nécessaire pour devenir professeur. Dans une lettre à Adorno, il s'explique longuement sur le "cas Habermas", qui a travaillé à l'Institut de recherche "sans jamais faire des expériences susceptibles d'étendre sa propre connaissance de la réalité sociale, hélas, sans même employer sa raison pour méditer le présent." Horkheimer enfonce le clou : "H. fait autant violence à la sociologie qu'à la philosophie". ${ }^{18} \mathrm{Si}$ Adorno semble vouloir modérer cette critique fondamentale, en garnissant cette lettre de quelques annotations manuscrites et d'une série de points d'interrogations, il tombe finalement d'accord sur l'essentiel, y compris la manière bancale d'aborder Marx, et partage la conclusion d'Horkheimer. Aujourd'hui, les amateurs de Habermas ne parviennent toujours pas à assimiler ou à digérer le désaccord qui est ici exprimé de manière aussi explicite que limpide, désaccord qu'Habermas a pourtant confirmé lui-même sur le plan théorique et politique. Axel Honneth ne veut y voir que du "pur ressentiment" de la part des fondateurs de la Théorie critique. Au cours de lignes suivantes apparaitra, au contraire, le caractère anticipateur, pertinent et lumineux de leur évaluation.

\section{Un Bad Godesberg intellectuel}

9 A la fin des années 1950, Habermas prépare donc son habilitation ailleurs, à Marbourg, au sujet du changement de l'espace public bourgeois, ce qui donnera son livre L'Espace public. ${ }^{19}$ Pendant ce temps, son parti, le SPD, prépare un nouveau programme politique, qui va signer sa rupture officielle avec le marxisme occidental, alors que son rejet du marxisme soviétique date de son refus majoritaire d'adhérer à l'Internationale communiste en 1920 (contrairement au socialisme français). L'enterrement de toute référence à Marx est acté lors du congrès de Bad Godesberg, en 1959. Alors que le directeur d'habilitation de Habermas, Wolfgang Abendroth, y défend une motion minoritaire pour la gauche marxienne et critique, l'écrit habermassien peut se lire comme une justification théorique du tournant programmatique officiel. Toutes les thématiques de ce congrès postmarxiste se retrouvent d'une certaine manière dans L'espace public habermassien : la centralité de l'Etat fédéral et national (contre l'internationalisme et la démocratie directe), la défense d'un libéralisme politique d'inspiration anglaise qui accorde la primauté à la propriété et à un marché juridiquement encadré (contre la critique du capitalisme tardif), le refus du syndicalisme ouvrier trop revendicatif (contre l'héritage 
conseilliste de la république de Weimar), le rejet global du marxisme et du droit naturel de la révolution française ou allemande (1791 et 1918-20). Le théoricien regrette dans ce livre que "la masse des non propriétaires" ait défiguré l'espace public bourgeois et libéral, à travers l'Etat social et les organisations de masse des gauches.

Muni de cette habilitation, Habermas va occuper un poste de professeur à Heidelberg, grâce au soutien de Hans Georg Gadamer qui va l'influencer intellectuellement. Le jeune professeur Habermas est ensuite nommé à l'Université de Francfort en 1963-64, mais en dehors de l'Institut qui a donné son nom journalistique à cette Ecole de Francfort. Même après la mort d'Horkheimer, qui l'en avait écarté, il refuse encore catégoriquement de rejoindre l'Institut de recherche sociale francfortois, qui a toujours été indépendant de la faculté. Rien ne serait donc plus faux que de prétendre que Habermas aurait pris la succession de Horkheimer, il a au contraire affiché la rupture la plus nette avec l'Institut co-fondé par Horkheimer. En 1971, Habermas déclare avoir rompu définitivement avec "l'Ecole de Francfort" dans une lettre à Herbert Marcuse. ${ }^{20} \mathrm{Ce}$ fait n'empêche pas la presse française de le présenter aujourd'hui encore comme un héritier d'Adorno et Horkheimer, et comme un représentant actuel de l'Ecole de Francfort, contre toute évidence. Nicolas Weill ${ }^{21}$ prétend encore que Habermas personnifie une seconde "Ecole de Francfort" dans Le Monde des Livres daté du 23 février 2018, là où le philosophe lui-même se place en rupture ouverte. Le journaliste le rencontre dans sa résidence bavaroise, ce qui suffit à indiquer la distance avec Francfort.

11 En réalité, Habermas n'a appartenu à l'Institut de recherche sociale de Francfort que pendant quelques années seulement, de 1956 à 1960, en qualité de boursier puis d'assistant, avant d'en être écarté, et il a toujours refusé d'y retourner par la suite. Habermas manifeste sa rupture définitive avec la Théorie critique en 1971. Pour couronner le tout, il refusa l'habilitation à Oskar Negt, un ancien doctorant et disciple direct d'Adorno qui a travaillé comme assistant d'Habermas au cours des années 1960, ce qui peut rétrospectivement apparaitre comme une sorte de revanche un peu mesquine, même si les deux intellectuels cohabitent aujourd'hui en paix. ${ }^{22}$ Negt est nommé professeur à la tête de l'institut de recherche de l'Université Hanovre en 1971, au moment précis où Habermas marque sa rupture définitive avec l'héritage adornien.

\section{La gauche réplique à Habermas}

Sur le plan politique, la direction du social-démocrate décide à cette même époque d'exclure l'organisation étudiante SDS qui affiche une ligne de gauche maintenue, malgré le congrès postmarxiste du SPD de 1959, suivie en 1963 de la dissolution forcée de l'association de soutien au SDS dont Abendroth et Adorno font partie. Habermas finit par rallier ce virage droitier du SPD, ce qui apparait publiquement en juin 1967 lorsqu'il insulte le porte parole du SDS, Rudi Dutschke, lors d'un congrès à Hanovre, en le traitant publiquement de "fasciste de gauche"23. En dépit de son excuse personnelle plus ou moins contrite, un groupe d'universitaires autour d'Oskar Negt publie alors un ouvrage collectif en soutien du SDS, intitulé La gauche réplique à Jürgen Habermas, ouvrage qui associe de grands noms de l'intelligentsia. ${ }^{24}$ Pendant ce cycle, qui va de 63 à 68 , le SPD participe au gouvernement fédéral sous un Chancelier de droite, Kurt Georg Kiesinger, ancien membre actif du parti national-socialiste, qui est finalement défait aux élections de 1969. Beate Klarsfeld avait auparavant sonné la charge, le giflant en pleine face lors d'un congrès du 
parti de droite CDU. Alors que Habermas traite le porte parole du mouvement étudiant de fasciste, son propre parti SPD maintient au pouvoir un ancien Nazi, nota bene.

Pendant ce temps, Theodor W. Adorno s'associe à son ancien doctorant Oskar Negt (un supporteur du SDS) pour parvenir à convaincre le dirigeant du principal syndicat, Otto Brenner, de lancer une campagne contre l'état d'urgence que le gouvernement Kiesinger s'apprête à appliquer avec le soutien du SPD. ${ }^{25}$ La coalition des intellectuels critiques, des jeunesses socialistes et des syndicats marqués par l'antifascisme parvient à bloquer cette voie, sans le concours d'Habermas. Ajoutons qu'Adorno loue les capacités critiques et les potentialités démocratiques du mouvement étudiant dans sa dernière lettre à Herbert Marcuse, comme en témoigne sa correspondance, malgré ses réserves envers le côté autoproclamé et activiste des jeunes porte-paroles. Sa critique n'a donc rien à voir avec le rejet habermassien du mouvement, comme Jean-Marie Vincent l'a remarqué à juste titre. 26

En écho à cette évolution de la République fédérale, la théorie habermassienne de la démocratie représentative et de l'espace public bourgeois ne conçoit aucune action collective à l'encontre de l'appareil de coercition étatique, comme le précise La théorie de l'agir communicationnel, ${ }^{27}$ où l'auteur dénonce toutes les formes de démocratie directe, socialiste ou participative. Il réduit ces approches à des vues purement moralisatrices : « Les tendances moralisatrices trouvent leur expression dans des idéaux d'autonomie ou de participation qui prédominent le plus souvent au sein de mouvements radical-démocratiques ou socialistes $» .^{28}$

15 Ces conceptions démocratiques portées par la gauche sont donc rejetées, car dérivées du droit naturel; elles ne disposeraient d'aucune base au sein des structures communicationnelles des sociétés contemporaines. Pareille approche démocratique serait complètement désuète, selon Habermas. Son incompréhension théorique, historique et sociologique de l'héritage vivant de la révolution française, en tant que révolution démocratique, peut donc logiquement s'agencer avec un soutien inconditionnel au président Macron sur le plan politique. La critique du caractère prétendument apolitique de la plèbe, de ces non-propriétaires qui n'entrent pas dans le schéma représentatif de l'espace public bourgeois, peut facilement s'accorder avec la dénonciation des grèves ouvrières ou des Gilets jaunes, justifiant le recours à la force pour rétablir un cadre institutionnel et représentatif qui borde le grand débat public.

\section{Présence de Gadamer, fantômes de Heidegger}

16 L'éloignement habermassien de l'Institut Francfort - entre 1960 et 71 - est soutenu par le professeur Gadamer, qui favorisa son arrivée à Heidelberg et restera une référence intellectuelle pour Habermas, en particulier pour sa manière de négocier un tournant langagier dans ses écrits. Dans l'après guerre, Hans Georg Gadamer, ancien membre de la ligue nazie des universitaires, a développé une herméneutique du langage fortement marquée par l'ontologie heidégérienne et ses thèses sur le langage.$^{29}$ Le livre de Gadamer Langage et vérité s'ouvre sur un chapitre issu d'une conférence de 1943 qui défend, comme le veut l'époque, l'Occident chrétien contre Marx, à travers la position restauratrice de Schelling, "appelé à combattre les effets politiquement et scientifiquement dangereux de la philosophie d'Hegel". ${ }^{30}$

Dans cette philosophie du langage, l'être se dit lui-même pour exister, en tant qu'acte d'auto-affirmation face au monde. Dans ce cadre ontologique, la société et les droits de 
l'Homme ne définissent pas cet être-là : l'Humanité n'existe pas. Le même livre montre comment Gadamer écarte catégoriquement de sa discussion des auteurs comme Benjamin, Lukacs et Adorno, en 1965 encore. Plus tard, Habermas s'emploie à traduire cette approche heideggérienne-gadamérienne, proche de sa thèse de doctorat, vers sa propre Théorie de l'agir communicationnel, en rupture ouverte avec la toutes les critiques formulées par Adorno dans le Jargon de l'authenticité et La dialectique négative. Il est inutile de souligner que la correspondance entre Horkheimer et Adorno foisonne de passages, où les deux chercheurs s'accordent pour dire qu'il s'agit de se méfier comme de la peste de ce Gadamer, qui se tient prêt à enfoncer un couteau dans leur dos. ${ }^{31}$ Une nouvelle fois, la critique plutôt dure de Horkheimer envers Habermas se concrétise. Car sa théorie langagière s'affranchit de toute expérience empirique, de tout apport des sciences sociales, pour relancer une ontologie qui retourne en définitive à Heidegger, à travers de multiples méandres. Dans La théorie de l'agir communicationnel, Habermas juge que la pensée d'Adorno - qui se déploie de manière dialectique à l'encontre de l'ontologie fermée de Heidegger - resterait bloquée par la métaphysique hégélienne, ce qui l'amène à ouvrir une perspective post-métaphysique qui ressemble étrangement à la position défendue par Heidegger et Gadamer dans l'après guerre.

Au cours des années 1970, Habermas élabore sa nouvelle théorie communicationnelle, qui se fonde sur une critique en règle de Kant, Marx, Lukacs, Horkheimer et Adorno en particulier. Le refus du "marxisme hégélien à la manière de Lukacs, Korsch, Adorno" apparait dès 1973 dans des conférences qui vont constituer son recueil Après Marx. ${ }^{32} \mathrm{Il}$ s'agit de préludes à La théorie de l'agir communicationnel, paru en 1980, qui est composé de deux grandes parties (édité en deux volumes dans sa version allemande). Le premier volet culmine dans une critique en règle de la pensée d'Adorno, sur une quarantaine de pages, alors que la seconde partie embraye sur une reconstruction de la sociologie traditionnelle de Durkheim et Weber, dont Adorno avait souligné les limites dans l'esprit de la Théorie critique. ${ }^{33}$ Il est ironique que Habermas ait reçu le prix Adorno pour cette oeuvre, qui cherche à contredire toutes les orientations du penseur francfortois. La persistance adornienne s'exprime bien davantage dans l'ouvrage de 1340 pages co-écrit par Negt et Kluge, Histoire et subjectivité rebelle, publié en $1980^{34}$, que Habermas ne mentionne pas. Il considère alors qu'il ne s'agite que d'explorations surréaliste sans épaisseur théorique,$^{35} \mathrm{ce}$ qui semble logique dans la mesure où il considère comme une vaine tentative l'oeuvre philosophique adornienne elle-même.

Tout en présentant les principaux textes de Horkheimer et Adorno, Habermas les annule progressivement dans son opus de 1981. Le point de chute du premier volume est un feu d'artifice en vue de l'explosion de la Théorie critique. ${ }^{36}$ Ainsi, la problématique philosophique majeure de La dialectique de la raison de 1944 qui culmine chez Adorno dans sa Dialectique négative de 1966 est présentée comme une aporie, pire, comme un simple exercice académique dénué d'objet et qui ne saurait aboutir. Ce n'est pas l'avis des chercheuses et chercheurs qui ont salué et relu l'ouvrage adornien à l'occasion du cinquantième anniversaire de sa publication, en 2016.

L'enquête empirique la plus étendue qui est signée par Adorno, Les études sur la personnalité autoritaire, ${ }^{37}$ est certes louée pour son apport antifasciste par Habermas en 1981, mais dépouillé de son soubassement psychanalytique, philosophique et sociologique, qui passe pour un sorte de folklore poussiéreux dans sa lecture. Il juge que ces recherches auraient été supplantées par des études politiques désormais courantes 
dans l'espace académique, sans voir que c'est leur originalité critique qui les différencie des sondages d'opinion et enquêtes statistiques mainstream.

De la même façon, toutes les études critiques sur les mass médias d'Adorno et de Benjamin, au sujet de la radio, du cinéma, de la télévision, etc., se trouvent retoquées par Habermas, en raison de leur approche radicalement critique envers la production marchande de produits stéréotypées et les arguments marxiens que cela sollicite, au sujet du fétichisme de la marchandise et la réification. Exit la critique de l'industrie de la culture, place à la sociologie traditionnelle.

L'une des idées centrales de la nouvelle théorie habermassiennne est que la communication se serait complètement affranchie des effets du capitalisme tardif et des rapports de classe, alors que les mass médias garantiraient le pluralisme démocratique. L'exemple de l'élection du président Trump invite à questionner pareille approche, dans la mesure où cet ancien acteur de téléréalité fut porté grâce aux mass médias et à la faveur de l'exploitation de 87 millions de profils personnels de l'entreprise médiatique Facebook, utilisés politiquement en 2016 par l'agence Cambridge Analytica au profit du candidat. Au départ, c'est le régime d'Hitler qui avait déployé les mass médias en tant qu'appareil de propagande, depuis le cinéma, en passant par la radio, jusqu'à une presse monopolisée, sous l'égide du ministère de la propagande nazie dont Rothacker fut l'un des chefs. La théorisation selon laquelle l'inspiration marxienne d'Adorno l'aurait conduit à une analyse trop critique du capitalisme et de ses effets sur les médias de masse apparaissent au mieux comme une naïveté dangereuse. Au vu de la grande crise capitaliste mondiale ouverte en 2008, Oskar Negt a commenté de manière critique ces conceptualisations qui apparaissent aujourd'hui fortement marqués par le contexte des années 1980 : «Rétrospectivement, les thèses habermassiennes qui postulent l'épuisement des théories du salariat, ainsi que la dissociation du système et du monde vécu, apparaissent comme des diagnostiques de circonstance, erronés ${ }^{38}$

En 1981, la critique adornienne du capitalisme tardif se trouve définitivement évacuée chez Habermas, à travers une valorisation de la théorie des systèmes, où les marchés et les administrations bureaucratiques nous sont présentés comme des domaines apolitiques, autorégulés, qui n'interfèrent pas avec les débats publics et politiques. Ici, les marchés et les flux de l'argent sont conçues comme un média, qui coexiste à côté des rouages para-étatiques, qu'il nomme le média du pouvoir, sans interférer avec un espace public dénué d'intérêts matériels. Laisser le marché et l'Etat en dehors de la discussion publique lettrée et éclairée semble garantir le règne de la raison. La crise financière mondiale de 2008 ou encore la surveillance en masse incontrôlée par des organismes comme la NSA ont souligné les limites de cette théorisation. Tout comme en 1929, des marchés capitalistes non régulés et des officines autoritaires de l'Etat sans contrôle menacent la démocratie, au lieu de garantir le bon fonctionnement d'un espace public et démocratique. Ni le marché capitaliste, ni les administrations publiques peuvent se passer d'un côntrôle démocratique direct par le peuple politique. Un autre exemple serait le rapport rendu par le Sénat français en février 2018, au sujet des dysfonctionnements graves et dérives privés dans l'organisation de la sécurité publique au sein de l'Elysée sous le président Macron. Les élus ne se sont pas montrés éblouis par les discours des hauts fonctionnaires qui ont tourné en roue libre sous l'autorité directe de l'exécutif. L'Etat autoritaire menace sans cesse la démocratie vivante, comme l'avait souligné Horkheimer dans ses écrits politiques, rédigés entre 1942 et $1970 .{ }^{39}$ 


\section{Le tournant bavarois}

24 Les efforts du penseur Habermas pour neutraliser la Théorie critique, qui culminent dans La théorie de l'agir communicationnel, se déploient dans le cadre d'un secteur de l'Institut Max Planck, situé dans une petite ville bavaroise, Starnberg. Il codirige cette section aux côtés de Richard von Weizsäcker, de 1971 jusqu'à la fermeture de cette partie de l'Institut en 1981. La nouvelle théorie communicationnelle s'écrit exactement entre ces deux dates, entre sa rupture avec la Théorie critique et la publication de son ouvrage majeur, qui achève l'épisode bavarois.

A la lecture de la biographie présentée par Müller-Doohm, il devient clair que l'échec intellectuel du programme lancé par Habermas et Weizsäcker, qui sera sanctionné par la fermeture de leur secteur bavarois, s'explique par des raisons que la nouvelle théorie communicationnelle élude soigneusement. Dénué d'un consensus méthodologique et transdisciplinaire qui serait appuyé sur des constats communs, les différents membres de l'Institut prétendent tous de posséder le meilleur argument, ce qui les rend incapables de dialoguer et de coopérer d'une manière productive. Le laboratoire s'effondre ainsi dans une forme de cacophonie intellectuelle, où les discours individuels priment sur les critères communs qui permettraient d'acter des résultats provisoires, de manière plus ou moins empirique. D'après la théorie communicationnelle de Habermas, la volonté de présenter le meilleur argument rationnel, au sein d'une délibération qui ne dépende pas de preuves empiriques ou sociologiques, devrait conduire à un dialogue fructueux, ouvrant un espace public dépourvu d'interventions extérieures. L'échec pratique de pareille approche communicationnelle souligne la faiblesse de la théorie nouvelle, et rappelle la justesse de la critique initiale de Horkheimer qui avait mis en relief l'absence de toute épreuve empirique dans les spéculations du jeune H. Souvenons-nous du constat critique que le jeune chercheur avait passé quatre années à ne tenir compte d'aucune expérience sociale à Francfort. A Starnberg, il a passé dix ans à formuler une théorie communicationnelle qui est contredite en pratique, au moment précis de sa publication. Depuis ce temps, Habermas n'est plus francfortois, mais bavarois.

Si Habermas ne s'est jamais entendu avec Horkheimer, il s'est bien accordé avec Richard von Weizsäcker pendant la période de l'Institut Max Planck. Il s'agit d'un homme de droite marquant. Avant de se repositionner au sein de la droite républicaine, le physicien Weizsäcker avait participé à l'équipe restreinte qui fut chargée de développer la bombe atomique sous la responsabilité directe d'Hitler, bien avant la fin de la guerre. Un autre physicien, Werner Heisenbourg, a témoigné par écrit du fait que Weizsäcker, membre du parti nazi, a déclaré vouloir "exterminer" tous ceux qui s'opposeraient à l'exécution de ce programme d'armement nucléaire (lettre à Elisabeth Heisenbourg, 14/10/1943). Devenu un dirigeant du parti de droite CDU après la guerre, aux côtés d'autres ex-nazis pénitents comme Kiesinger, Weizsäcker s'est publiquement distancié des crimes contre l'humanité commis sous Hitler, en se prononçant pour le pacifisme, alors qu'Habermas avait manifesté contre la menace d'une guerre nucléaire lorsqu'il était un jeune chercheur. Cette prise de position pacifiste doit être soulignée, dans la mesure où elle marque une nette rupture historique avec le bellicisme des Jeunesses hitlériennes, mais force est de constater qu'elle intervient après la bataille, bien après la défaite militaire des nazis. Cette morale pacifiste ne tiendra cependant pas très longtemps. 


\section{Otan, Vatican, Macron}

27 Au cours des années 1990, Habermas va se distancier à nouveau très clairement du pacifisme, d'un point de vue philosophique et politique. Lors de la chute du mur de Berlin et de l'Union soviétique, Habermas déclare que la nouvelle situation historique le rend plus conservateur encore.

Parmi les illustrations pratiques de la nouvelle profession de foi plutôt conservatrice d'Habermas comptent son dialogue public avec l'archevêque de Cologne à la réputation particulièrement réactionnaire, M. Ratzinger, lui aussi un ancien des Jeunesses hitlériennes, qui deviendra plus tard Benoit, le premier pape démissionnaire de l'histoire catholique. Une nouvelle fois, le thème onthéologique refait surface.

Un autre fait d'armes de cette période habermassienne est sa prise de position en faveur des bombardements de la Serbie par l'Allemagne dans le seul cadre militaire de l'Otan (Die Zeit, 29/4/1999), alors que des intellectuels de gauche autour de Pierre Bourdieu militent pour une paix juste dans les Balkans. Le penseur bavarois emboite le pas au gouvernement social-démocrate qui impose ces frappes, sous le Chancelier Schröder. Dans L'intégration républicaine ${ }^{40}$ Habermas avait déjà vilipendé le pacifisme juridique d'Emmanuel Kant, en recourant aux écrits du juriste Carl Schmitt, un membre du parti nazi qui avait soutenu Hitler depuis le 1er mai 1933, avant de justifier la guerre d'agression jusqu'en 1945 sur le plan théorique et idéologique. A la page 162 de son livre, Habermas n'hésite pas à souligner que la critique schmittienne du pacifisme juridique a eu "une remarquable efficience historique" (nous parlons de la seconde guerre mondiale), avant d'actualiser cette approche. Le fait qu'Habermas soutienne le bombardement de la Serbie, en l'absence de tout mandat international de l'ONU, peut aussi éclairer les limites du modèle abstrait de l'éthique de la discussion qu'il promeut. Les sociologies des médias plus critiques cherchent au contraire à montrer que des intérêts sociaux non-dits et des rapports de domination de toute sorte interviennent dans toute discussion publique. Le tournant linguistique habermassien, qui croit que tout rapport entre les sujets et objets peut s'harmoniser dans le langage, a favorisé une compréhension du social qui serait dénué de domination, de conflits et de rapports de classe. Bien entendu, une telle vision peut se montrer vulnérable à la communication politique mainstream, si ce n'est à la propagande. Est-ce le fruit du pur hasard que Habermas soutient de manière aussi récurrente les positions gouvernementales les plus discutables des chefs d'Etat, de Kiesinger à Macron?

Les années 2000 montrèrent Habermas comme animateur du club de réflexion européen de Dominique Strauss Kahn, dans la perspective de la mise en application de la stratégie de Lisbonne de l'Union européenne, qui prétend avancer vers une société du savoir et de plein emploi, d'un genre post-industriel. Sur le plan théorique, Adorno avait signalé que la question n'est pas de savoir si la société serait industrielle ou non, mais si la critique marxienne du capitalisme reste pertinente ${ }^{41}$ La réponse est oui pour Adorno, le président de la société de sociologie allemande. Encore une fois, Habermas prolonge un élan discursif et théorique qui se situe loin de la Théorie critique.

Lors du référendum de mai 2005 qui porte sur un projet de constitution européenne, synthétisant l'ensemble des traités de teneur néolibérale, Habermas intervient dans Le Monde (3/5/2005) pour exhorter les citoyens à approuver ce cadre. D'un point de vue philosophique, Habermas défend alors le droit positif, l'identité nationale et l'ordre établi 
contre le droit naturel de la révolution française. L'absence de constituante pour fonder une constitution de l'Europe ne le gène pas, dans la mesure où il refuse catégoriquement toutes les formes de démocratie radicale ou participative. Politiquement, Habermas soutient la seconde gauche et sa troisième voie, mais son candidat de coeur, DSK, sort du jeu politique avant la présidentielle française de 2012, à cause d'une série de dérives. François Hollande se contente de citer Habermas dans son livre de campagne électorale, où le futur président ne cite aucun autre philosophe. Avec la candidature d'Emmanuel Macron en 2017, Habermas trouve enfin une figure d'identification politique pleine et entière, à la droite de la social-démocratie européenne. Le philosophe se démarque alors pour la première fois politiquement de la direction de son propre parti, le SPD, dont la direction a clairement appelé à voter pour Benoit Hamon, alors candidat du PS. Habermas se déclare, lui, supporteur d'Emmanuel Macron avant le premier tour des présidentielles et se dit ébloui. Adorno et Horkheimer l'avaient mis en garde contre la connexion aveuglante qui émane du capitalisme global et de ses représentations fétichistes. La critique scientifique que Horkheimer avait adressé au jeune candidat Habermas se confirme encore. Il peut néanmoins paraitre surprenant que le théoricien de l'agir communicationnel adhère sans réserve à un tel type de personnification plébiscitaire, plus proche du bonapartisme que du libéralisme politique. Ce paradoxe s'explique si l'on regarde de plus près sa construction philosophique.

\section{Un hégélien de droite en dehors du temps}

Le penchant pour des solutions et des discours d'autorité se manifeste nettement dans le livre L'intégration républicaine $e^{42}$, où Habermas cherche à cerner les contours d'une "identité nationale", et à évacuer définitivement la philosophie du droit naturel qui est intervenue dans la définition de la première constitution de la révolution française de 1793. Habermas voudrait clore le mouvement continu pour des droits sociaux égalitaires, en postulant qu'il faudrait se contenter de la déclaration initiale des droits de l'Homme de 1791, qui n'est en réalité que le préambule d'une constitution révolutionnaire qui fut bel et bien adoptée mais jamais appliquée. Cette déclaration permet, selon lui, de penser une positivation du droit qui annoncerait une constitution européenne, assurant une sorte de droit positif des propriétaires. Le fait est que les philosophes anglais comme John Locke, qu'Habermas invoque souvent, défendent eux le droit naturel des citoyens, nés libres et égaux, contre le droit positif. Kant moque à son tour ce type de droit comme une sorte de fatras de circonstance, le comparant à une belle tête dépourvue de tout cerveau ${ }^{43}$ Il faut admettre que les philosophes du droit naturel défendent sans exception la propriété comme un droit fondamental, bien avant l'émergence des mouvements socialistes. Habermas ne se contente pas de revenir à ce point de départ ancien, puisqu'il cherche à rétablir un droit positif et traditionnel qui implique des inégalités de principe. Au fait, le principe constitutionnel du droit naturel s'incarne dans la première constitution de la république, adoptée en 1793, qui prolonge la proclamation des droits de l'homme au sens d'une égalité intégrale des droits entre tous les citoyens. Cette constitution ne fut pas positivée, mais déniée et supprimée par les thermidoriens, puis par l'empire bonapartiste, qui mit fin à la république, rétablissant l'esclavage. En rejetant le droit naturel de la révolution, sa théorisation cohérente chez Kant, ses prolongations chez Marx et dans la Théorie critique, Habermas s'inscrit ainsi dans une filiation théorique qui abandonne l'égalité de principe et écarte la participation démocratique directe des "nonpropriétaires". Cet aspect théorique recoupe un important clivage historique, entre 
continuateurs de la révolution française et opposants à la révolution démocratique. Dans la philosophie allemande, devenue internationale lors de la révolution de 1848 , ce clivage se manifeste dans l'opposition entre hégéliens de gauche et de droite. Les hégéliens et hégéliennes de gauche, dont Otto Bauer, Karl Marx ou la philosophe féministe Louise Dittmer font partie, veulent appliquer le droit de la révolution française à l'échelle internationale, par la révolution démocratique, alors que les hégéliens de droite cherchent à conserver l'Etat impérial, en le dotant d'un droit constitutionnel pérenne (par exemple Georg Gabler, Carl Göschel).

33 Ce courant de droite prolonge la théorie du droit de Hegel, qui de vivant était passé du soutien à la révolution française au ralliement à Napoléon Bonaparte, puis à l'idée d'un Etat impérial constitutionnel allemand, dépourvu de participation démocratique. Ce modèle hégélien, a-démocratique, fonde la souveraineté de l'Etat sur la propriété, la famille bourgeoise, elle-même liée au contrat du mariage, ainsi que sur les conventions des corporations de métier, sur les administrations ou bureaucraties non-élues (les corps intermédiaires dans le langage hégélien ou impérial). Voilà la théorie du droit constitutionnel que Hegel livre en $1821 .^{44}$

Cette théorie politique hégélienne, qui est durement critiquée par Marx, lance aussi l'idée d'un "patriotisme constitutionnel", qui est devenu un leitmotiv de Habermas. Tous les fondamentaux de la conception habermassienne sont présents dans cette version hégélienne conservatrice, qui deviendra l'apanage des hégéliens de droite : la souveraineté de l'Etat national (plutôt que la souveraineté démocratique absolue du demos); la sacralisation de la propriété et l'exclusion des non-propriétaires de l'espace public bourgeois; l'indifférence au féminisme; la limitation du syndicalisme à une représentation apolitique et corporative en tant que corps intermédiaire; la limitation de la représentation politique à un vote qui ne change pas la nature du pouvoir souverain; l'interdiction de toute démocratie directe, participative ou socialiste. Voilà l'esprit de la loi travail et des ordonnances Macron de 2016-17, qui cherchent à limiter le pouvoir interprofessionnel, revendicatif et solidaire des syndicats, pour les réduire à des auxiliaires dans la négociation d'accords d'entreprise. Le mouvement ouvrier global se voit ramené aux corporations qui garantissent le bon ordre de la société bourgeoise. Aussi, la valorisation de la société civile et du débat public entre citoyens-bourgeois qui ne trouble pas la souveraineté étatique, conçue par Hegel, émaille l'ensemble des écrits habermassiens. Partie d'une valorisation du contre-révolutionnaire Schelling, sa pensée récuse le droit naturel de la révolution française, cherche à réfuter Kant, à neutraliser les hégéliens de gauche, à contrer Marx et la Théorie critique, un courant qu'il identifie à juste titre comme une prolongation de cet élan intellectuel.

35 A mon sens, Habermas cherche à sauver une certaine version de la théorie du droit hégélienne, dans sa formulation de 1821, qui est conservatrice, droitière dans sa réception et ambivalente dans sa filiation historique. Identité nationale, patriotisme constitutionnel, exclusion des non-propriétaires, des prolétaires, du féminisme, du socialisme, refus de la démocratie directe. Ce modèle hégélien, qui se retrouve relancé chez les hégéliens de droite après sa mort, semble être transposé à l'ordre républicain de l'Etat français ou allemand chez Habermas. Il ne faudrait pas croire que je projette une interprétation hégélienne lointaine sur une théorie sociale plus contemporaine, puisque Habermas se charge lui-même de situer sa position en lien avec ce contexte philosophique ancien. Il définit théoriquement la position des hégéliens de droite dans un paragraphe qui ressemble à un autoportrait intellectuel: "Pour les hégéliens de droite (...) 
c'est la rationalité d'entendement érigée en absolu qui s'exprime désormais à travers l'exaltation des idées socialistes; et pour faire pièce à ces faux critiques, il n'y a qu'une seule possibilité : imposer l'intelligence métacritique des philosophes." ${ }^{45}$ Le philosophe surplombe ainsi le débat démocratique, tout comme l'Etat prime sur le débat politique qui oppose gauches et droites. Dans ce texte, Habermas peine à tracer une frontière conceptuelle claire entre un Hegel impérial, un Schelling contre-révolutionnaire, et des théoriciens issus du nationalsocialisme qui tâchent de se réinsérer dans le jeu philosophique dans l'après guerre, à l'instar de Heidegger et de Gadamer. Pour bien saisir la ligne de crête théorique habermassienne sur laquelle se tient perché l'intellectuel post-francfortois, il convient de retracer le versant hégélien droitier à grands traits, rapides mais incisifs. Les hégéliens de droite sont définis dans la période qui va de la mort de Hegel en 1831 à la révolution européenne de 1848, ils veulent éviter une contre-révolution royaliste violente et proposent une constitution impériale qui garantisse les droits civiques, sans chercher à établir la démocratie.

Précisons ici un fait historique : la révolution européenne de 1848 donne lieu, en Allemagne, à une assemblée constituante qui se réunit à Francfort, ou les délégués de la gauche revendiquent une constitution républicaine (inspiré de la première république française), où la droite veut une monarchie constitutionnelle qui maintienne le Reich (l'empire), et où le centre hésite. Ce centre allemand finit par se rallier à la droite et à l'armée Prusse pro-impériale, contre les manifestations de rue pro-républicaines. Bien entendu, la contre-révolution royaliste se situe dès le départ en dehors de la constituante francfortoise, mais c'est en son for que se forge la décision de rendre le pouvoir aux rois. La frontière politique entre les délégués de droite dans l'assemblée constituante et la position contre-révolutionnaire de la réaction royaliste qui refuse d'y siéger n'est donc pas nette et catégorique. Ce flou politique resurgit sous une autre forme en 1933, lorsque la droite républicaine s'allie à l'extrême droite de Hitler pour lui confier le pouvoir de manière apparemment légale et constitutionnelle. Sur le plan politique, cela s'exprime dans le passage de l'état d'exception constitutionnel de la république de Weimar à sa suspension totale, un passage à l'autoritarisme qui fut entièrement théorisé par Carl Schmitt, juriste d'Hitler, et soutenu par une philosophie post-hégélienne qui conteste le droit naturel, fournie par Heidegger.

En Allemagne, les intellectuels contre-révolutionnaires ont toujours cherché à bannir l'héritage démocratique de la révolution française, et à rétablir la souveraineté impériale, qui peut tout à fait intégrer la société bourgeoise, comme l'empire bonapartiste l'a montré. Rosa Luxembourg, Walter Benjamin, George Politzer, Pierre Bourdieu, Theodor W. Adorno, ou Zveen Sternhell ont montré, tous à leur manière, que cette impulsion impériale-réactionnaire de la droite intellectuelle se transforme immédiatement en contre-révolution violente ${ }^{46}$, dès que la révolution allemande des conseils de novembre 1918 parvient à renverser l'empire. Le principe de souveraineté impériale venait de se manifester dans la conduite de la guerre mondiale, appuyé sur un "patriotisme constitutionnel" de l'empereur Guillaume qui voulait faire taire les oppositions de gauche. Dès la proclamation de la république, par les gauches socialistes et conseillistes, et la convocation d'une constituante démocratique (incluant les travailleurs et les femmes, sans discrimination religieuse aucune), l'intelligentsia impérialiste et cléricale se fait contre-révolutionnaire et pro-impériale. L'assassinat de Rosa Luxembourg, militante de gauche, intellectuelle, femme politique, d'origine juive et de conviction athéiste, qui milita contre la guerre, symbolise toute la violence de ce mouvement impérialiste, qui 
reprend à son compte la propagande au sujet d'un prétendu "judéo-bolchévisme". Ce discours paranoïaque émane des cercles aristocratiques de l'armée impériale défaite, dès $1918^{47}$, avant d'être systématisé par la propagande nazie. ${ }^{48}$

Le courant intellectuel impérialiste le plus radicalisé, allemand et autrichien, évolue ensuite vers le national-socialisme, poussant jusqu'à l'extrême et jusqu'à la volonté exterminatrice les thèses les plus répandues de la restauration impériale. Partant des penchants les plus droitiers de l'héritage bonapartiste de Hegel, de l'anti-hégélianisme de Schelling et de la posture contre-révolutionnaire d'un Fichte tardif, ce courant évolue vers une définition absolue de l'autorité et de l'identité nationale. Cette définition autoritaire s'incarne finalement dans une relance fanatique de l'empire qui se prétend le troisième empire allemand, le Troisième Reich dans la propagande hitlérienne. Pareil projet impérialiste, anti-démocratique, raciste, violemment antisémite, anti-slave, antirrom, qui se montre aussi anti-féministe et homophobe, est porté par des intellectuels comme Martin Heidegger, Carl Schmitt, Ernst Jünger, et en seconde ligne par des idéologues comme Alfred Rosenberg, Hans Georg Gadamer ou Erich Rothacker (le directeur de thèse de Habermas). Tous ont oeuvré en faveur de la construction symbolique du national-socialisme, avant même d'adhérer formellement à son chef suprême en 1933. Le chef est tombé, mais leurs théorisations ne permettent toujours pas de rompre avec les principes autoritaires et identitaires qui les fondent.

\section{A propos de l'antifascisme}

Bien entendu, Habermas veut se démarquer explicitement de la filiation antirépublicaine, par son refus politique de l'extrême droite allemande, européenne. A côté de son faible pour le versant conservateur d'un Hegel - qui se voulait lui-même patriote constitutionnel -, cela va de pair avec la reprise habermassienne du positivisme étatique de Durkheim, qui était dreyfusard, au nom de l'Etat de droit. ${ }^{49} \mathrm{~S}^{\prime} \mathrm{y}$ ajoute la théorie politique de Max Weber, que Habermas conçoit comme un conservateur authentique (erzkonservativ). ${ }^{50}$ Ces traditions ont aussi en commun d'être explicitement opposés à Marx et aux socialismes, un trait constant dans la pensée habemassienne, excepté sa brève période francfortoise. Il est vrai que Habermas a connu le national-socialisme de manière intime, dans sa jeunesse et pendant ses études, ce qui le rend parfaitement capable de percevoir les tentatives de relance propagandistes, même sous la forme de discours raffinés, comme on l'a vu pour Nolte et Sloterdijk.

Pareille position fait de Habermas un théoricien presque idéal du discours et du pouvoir macronien, élu par contraste au Front national en 2016, mais compatible avec les traditions libérales, bonapartistes ou post-vichystes de la droite française, qui s'accordent avec la constitution de la 5ème république et ses postures autoritaires, avec son appareil de répression violent qui alerte jusqu'aux Nations Unies et à la direction des droits humains du Conseil de l'Europe.

41 En France, le patriotisme constitutionnel signifie en pratique le ralliement à un pouvoir présidentiel élu, mais placé à l'abri de tout contrôle démocratique, dans le droit fil de la vision du Hegel de 1821. Tout se passe comme si Habermas venait à légitimer philosophiquement les positions des hégéliens de droite et de la droite parlementaire de Francfort de 1849, contre la démocratie directe et l'impulsion de la révolution française, contre Marx et contre le socialisme international. 

discours post-hégélien, une formule fourre-tout qui embrasse tout ce qui vient après la mort d'Hegel en 1831. L'affirmation post-hégélienne débouche sur une énumération où il égrène pêle-mêle Marx, Heidegger, Derrida, l'Ecole de Francfort, etc., sans distinction aucune. Pareille énumération verse facilement dans l'anachronisme, faisant disparaitre tout contexte historique précis. L'hégélianisme, le marxisme, le fascisme, tout se trouve associé et mélangé dans une critique anhistorique. Le choix habermassien de partir de l'année 1831 - la mort d'Hegel - pour aborder les théories post-hégéliennes, occulte la spécificité de la période de 1830 à 1848 , le Vormärz, qui inclut la formation des hégéliens de gauche, la rédaction du Manifeste communiste, l'émergence du mouvement démocratique européen et l'effondrement du discours impérial. D'ailleurs, lorsque Habermas définit Dutschke et le courant socialiste radical qui se déploie avant 1968 de "fasciste de gauche", il le compare précisément au socialisme utopique qui travaille le moment 1848, dans la même intervention, dans une même phrase. ${ }^{51}$ Habermas s'est excusé d'avoir diffamé Dutschke, mais il maintient de toute évidence le dénigrement du socialisme historique, utopique ou marxien que son argumentation implique. En ce sens, la question reste de savoir si le discours postmarxiste de Habermas concerne toute la postérité de Marx depuis son décès, ou la période depuis le virage postmarxiste du SPD en 1959, ou alors s'il doit être daté de son propre tournant antimarxiste qui se manifeste avec son Après Marx en 1978? En tout cas, la période qui relance la publication et la réception des écrits de Marx débute avec la crise mondiale de 2008, ce qui fait que le postpost marxisme a commencé il y a dix ans. Aujourd'hui, Google scolar fait état de plus d'un millions de livres ou articles qui citent Marx : 1.260 .000 références, et la tendance est à la hausse depuis des années.

43 A travers son anti-socialisme intellectuel, doublé d'un refus du droit naturel de la révolution française, Habermas se rapproche de facto des positions anti-totalitaires et contre-révolutionnaires de François Furet, à l'égard de la révolution française et de la gauche marxienne ou francfortoise. Fidèle à sa ligne de démarcation idéologique, Habermas refuse dans le même temps la version allemande, radicalisée, de pareille approche que présenta l'historien révisionniste Ernst Nolte, que Habermas dénonça à juste titre dans la presse (Die Zeit, 11/7/86). Nolte voulait faire passer le nazisme et même son programme d'extermination tout entier pour une simple réaction défensive, face au communisme de l'entre-deux guerres. La différence avec l'approche de Furet demeure subtile, ou faible, d'après l'historien Sternhell, elle n'en reste pas moins significative. ${ }^{52}$ Cela montre que Habermas refuse autant les pistes ouvertement néofascistes qu'il repousse la révolution française et sa filiation marxienne ou francfortoise, se plaçant au centre de tout. L'antimarxisme militant de Furet semble tolérable, le néofascisme de Nolte est contestable. Le discours heideggérien de Gadamer oui, les pamphlets heideggériens de Sloterdijk non - l'un se contente d'écarter les héritages critiques de la gauche, tandis que le second singe désormais le discours de Goebbels à l'encontre de la "presse du mensonge", en évoquant un mystérieux "éther du mensonge"..$^{53}$ Pour Habermas, il est totalement exclu de revenir à la propagande nazie de Rothacker d'avant 1945. Cela semble une ligne minimale, mais néanmoins importante, au vu de la résurgence de l'extrême droite et de ses dérives éditoriales.

Voici certaines affinités de ce savant équilibre avec la position macronienne : un républicanisme conservateur, tourné en même temps contre les mouvements démocratiques directs, la gauche historique, et contre les formes idéologiques les plus 
affirmées de l'extrême droite. Reste un centre-droit adossé à l'Etat régalien, appuyé sur une médiatisation massive. L'éloge présidentiel de l'Europe en opposition au nationalisme, prononcé en Sorbonne (26/09/2017), qui avait plu à Habermas, doit désormais cohabiter avec un hommage présidentiel au maréchal Pétain, ce "grand militaire" (7/11/2018), qui en réalité fut déchu de ce titre pour trahison par la république. Le dispositif global est appuyé sur la pérennisation légale de l'état d'urgence dont le principe fut adopté lors de la guerre d'Algérie, une guerre coloniale. Au fond, ces compositions concernent différents courants de droite plus ou moins bonapartistes, postpétainistes ou républicains, et non pas les conceptions historiques de la gauche dont Kant, Marx et la Théorie critique font partie. Habermas couvre désormais un champ intellectuel partial, diminué, de moins en moins représentatif du débat public au sens large.

Dans ce contexte, il semble complètement exagéré d'identifier le discours républicain de Habermas à la riche tradition de Lumières, dans la mesure où l'auteur se distancie des philosophes du droit naturel, depuis Kant et la révolution française, dont la filiation est reprise par Marx, Benjamin, Bloch ou Adorno, qui prend Kant comme point de départ d'une dialectique de la Aufklärung pour en sauvegarder le principe initial. Oskar Negt ou Nancy Fraser perpétuent cet héritage à leur manière, dont les traces rayonnent sur les cinq continents à travers les jeunes générations. Les lumières de Habermas vont, elles, de pair avec l'éblouissement pour la figure d'autorité d'Emmanuel Macron.

\section{Une théorie juste pour une société fausse}

La position habermassienne, hier dénuée d'une analyse empirique solide, aujourd'hui fondée sur une adhésion publique à la position jupitérienne du président, semble singulièrement mal appropriée pour encourager des compréhensions et des pratiques démocratiques qui cherchent à sortir du discours de l'identité nationale. Cela signifie que les ressources critiques sont à rechercher en dehors de cette filiation théorique, dont les limites ont été clairement désignées par des figures aussi avisées que Max Horkheimer. Les intellectuels francfortois n'ont été aveuglé ou ébloui par aucune figure charismatique, ni par Bonaparte, ni par Hitler, ni par Kiesinger, ni par le Général de Gaulle et encore moins par un haut fonctionnaire, ancien banquier qui se rêve en Jupiter à l'Elysée. Adorno y aurait probablement vu une énième variation de la personnalité autoritaire qui n'a nullement besoin de s'identifier au fascisme pour exister. La Théorie critique dit qu'il est vain de vouloir résister aux formes autoritaires ou néofascistes sans remettre en question les conditions sociales de leur émergence. "Celui qui ne veut pas parler du capitalisme doit se taire au sujet du fascisme", affirmait Horkheimer à juste titre. ${ }^{54}$

Adorno avait désigné les statistiques et sondages d'opinion comme l'image juste d'une société fausse. La théorie de Habermas peut se lire, elle, comme l'image juste d'une politique fausse, ou comme la communication réussie d'un modèle qui est en échec historique. 


\section{NOTES}

1. K. Marx, Das philosophische Manifest der historischen Rechtsschule (1842) , MEW, 1, Dietz,1957, p.80.

2. R. Dely, Strauss Kahn discret avec Schröder, Libération, 6/9/2005.

3. S. Müller Doohm, Jürgen Habermas, Gallimard, 2018.

4. I. Kant, Kriecherei (L'esprit du grouillot) in : Metaphysik der Sitten, Suhrkamp, 1977, p.569.

5. H. Boberach, Jugend unter Hitler, Gondrom Verlag, 1990.

6. U. Thamer, Der Nationalsozialismus, Reclam, 2002, p.269.

7. L'ensemble des CV des intellectuels compromis dans le national-socialisme est documenté par

E. Klee, Personenlexikon des Dritten Reiches, Nikol, 2016.

8. E. Faye, Martin Heidegger : l'introduction du nazisme dans la philosophie. Autour des séminaires de 1933-1935, Le livre de poche, 2016.

9. V. Farias, Heidegger et le national-socialisme, Verdier, 1987.

10. Les sympathies de Heidegger pour les SA sont documentées par Farias, op.cit.

11. La police, l'armée, les SS et la Chancellerie d'Hitler se liguent et isolent définitivement Röhm à ce moment précis, comme les livres d'histoire le montrent : U. Thamer, Der Nationalsozialismus, Reclam, 2002, pp.170-171.

12. Voir p.ex. F. Rastier, Heidegger, Messie antisémite, Le bord de l'eau, 2018.

13. Cette phrase devrait figurer dans Heidegger, Gesamtausgabe, vol.69 - Rastier, op.cit., le précise à l'appui d'une note de l'éditeur P.Trawny.

14. Lettre de Marcuse à Heidegger depuis NYC, daté du 28 aout 1947.

15. Il semble absurde et anachronique de présenter cette position heideggérienne intégrale de 1953, qui n'est plus revendiquée par Habermas, comme un prétendu point d'équilibre dans la discussion actuelle sur la pénétration antisémite et nazie de l'oeuvre de Heidegger, comme tente de le faire maladroitement le journaliste N. Weill dans son essai Mysticisme du ressentiment, Editions CNRS, 2018. Voir l'état de l'art sur le sujet : F. Rastier, directeur de recherche CNRS, Heidegger, messie antisémite, Le borde de l'eau, 2017.

16. Cours de 1936 de Heidegger sur Schelling : M. Heidegger, Gesamtausgabe, vol. 42, Klostermann, 1988.

17. Pour la réception national-socialiste de Schelling voir p.ex. Kurt Hildebrandt, Schelling und die deutsche Bewegung, Reclam, 1942.

18. Adorno/Horkheimer, Briefwechsel1950-69, tome IV, Suhrkamp, p.508 (ed. française Correspondance IV, Klincksiek, 2016, pp. 386-399).

19. J. Habermas, L'espace public, Payot, 1994 ou : Habermas, Strukturwandel der bürgerlichen Öffentlichkeit, Suhrkamp, 1962.

20. S. Müller Doohm, Jürgen Habermas, Gallimard, 2018.

21. Le journaliste affirme à la fois que Habermas serait parti à Francfort car trop à gauche aux yeux des fondateurs, tout en constatant qu'il y était opposé à l'aile gauche de la Théorie critique. Bien entendu, le post-heideggérien Habermas se place à la droite de Horkheimer et Adorno, qui discutaient les contours d'un nouveau Manifeste communiste du genre marxien avant son départ : Horkheimer/Adorno, Towards a New Manifesto, Verso Books, 2012.

22. O. Negt, R. Stollmann, «Entretien », in Schulten/Stolmann : Der Maulwurf kennt kein System, Transcript, 2005, http://www.transcript-verlag.de/ts273/ts273_1.pdf

23. Intervention de Habermas retranscrite dans Rudi Dutschke, Geschichte ist machbar, Wagenbach, 1992, pp. 82-84. 
24. O. Negt (dir.), Die Linke antwortet Jürgen Habermas, éd. EVA, 1968.

25. O. Negt, Achtundsechzig, Steidl, 1995; Adorno, Gegen die Notstandsgesetze, in : Vermischte Schriften, II, stw, 1997, p.396

26. J.M. Vincent, Adorno contre Habermas et au-delà, Futur antérieur, ré-édité dans le cadre du présent $n .22$ de la revue Variations.

27. J. Habermas, La théorie de l'agir communicationnel, Fayard, Paris, 1987.

28. J. Habermas, Theorie des kommunikativen Handelns, Suhrkamp, 1981, p.520 (nous traduisons).

29. M. Heidegger, Zum Wesen der Sprache, Klostermann, 2010.

30. H. Gadamer, Langage et vérité, Gallimard, 1995, p.59; Gadamer, Les chemins d'Heidegger, Vrin, 2002.

31. Adorno/Horkheimer, op.cit. (voir index des noms cités).

32. Le texte allemand qui sert de base à Après Marx a pour titre plus ambitieux : La reconstruction du matérialisme historique. J. Habermas, Rekonstruktion des historischen Materialismus, Suhrkamp, 1976, p.158.

33. Adorno, Le conflit des sociologies, Payot, 2016; A. Neumann, Dissonances d'Adorno in : Après Habermas, Delga, pp.66-79.

34. Negt/Kluge, Geschichte und Eigensinn (1980), Steidl, 2018. Voir F. Jameson, Histoire et subjectivité rebelle, Variations n.21 https://journals.openedition.org/variations/896; O. Negt, L'espace public oppositionnel, Payot, 2007.

35. Voir O.Negt, "Entretien", in Schulten / Stollmann, Der Maulwurf kennt kein System, Transcript, 2005, http://www.transcript-verlag.de/ts273/ts273_1.pdf

36. J. Habermas, Theorie des kommunikativen Handelns, Band 1, Suhrkamp, 1981, pp. 489-534.

37. Adorno, Les études sur la personnalité autoritaire, Allia, 2007.

38. O. Negt, entretien accordé à la revue Sozialismus, VSA, 2011, p.56.

39. M. Horkheimer, Gesellschaft im Übergang, Fischer, 1972.

40. J. Habermas, L'intégration républicaine, Fayard, 1998.

41. Adorno, Société industrielle ou capitalisme tardif ? (1968) in : Société, Payot, 2012.

42. J. Habermas, L'intégration républicaine, Fayard, 1998.

43. Eine bloß empirische Rechtslehre ist (wie der hölzerne Kopf in Phädrus' Fabel) ein Kopf, der schön sein mag, nur schade! dass er kein Gehirn hat : E. Kant, Rechtslehre, VI, p.230.

44. J.W. Hegel, Grundriss der Philosophie des Rechts, Meiner, (1821) 1921.

45. J. Habermas, "Hégéliens de gauche, hégéliens de droite, Fichte" in : Le discours philosophique de la modernité, Gallimard, 1988, p.68.

46. Th. W. Adorno, Dialektik und Ontologie, Suhrkamp, (1961) 2010; Adorno, Jargon de l'authenticité, Payot, (1964) 1989; Adorno, Dialectique négative, Payot, (1966) 2003; W. Benjamin, Walter, Sur le concept d'histoire, Payot, (1940) 2017; P. Bourdieu, L'ontologie politique de Martin Heidegger, Minuit, 1975; V. Farias, Victor, Heidegger et le nazisme, Verdier, 1987; E. Faye, Emmanuel, L'introduction du nazisme dans la philosophie, Albin Michel, 2005.

47. W. Wette, Les crimes de la Wehrmacht, Perrin, 2009.

48. G. Politizer, Révolution et contre-révolution au 20ème siècle (1940), Editions critiques, 2017.

49. Voir E. Durkheim, Le socialisme, 1928 :

http://classiques.uqac.ca/classiques/Durkheim_emile/le_socialisme/le_socialisme.html

50. J. Habermas, Theorie des kommunikativen Handelns, stw, I, 1981, p.497. En réalité, Weber évolua vers le libéralisme de gauche et discuta amicalement avec L. Trotsky et G. Lukacs, voir J.M. Vincent, Max Weber ou la démocratie inachevée, Le Félin, 2009.

51. R. Dutschke, op.cit., p.82.

52. Zveen Sternhell, Les anti-Lumières, Gallimard, 2006, pp.774-794.

53. P. Sloterdijk, Es gibt keine moralische Pflicht zur Selbstzerstörung, magazine Cicero, 1/2016. 
54. Max Horkheimer, Die Juden und Europa (1939), in : Gesammelte Werke vol.4, Suhrkamp, 1988, p.308.

INDEX

Mots-clés : Theorie de l'agir communicationnel, espace public, modèles critiques, Jürgen Habermas, Max Horkheimer, Theodor W. Adorno, Oskar Negt

\section{AUTEUR}

\section{ALEXANDER NEUMANN}

Professeur des Universités, Paris 8 